\title{
A reverse form of Linburg-Comstock variation with comments on its etiology and demonstration of interactive 3D portable document format
}

\author{
Wijittra Prasatkaew ${ }^{1,2} \cdot$ Nutmethee Kruepunga $^{1,2} \cdot$ Laphatrada Yurasakpong $^{1,2} \cdot$ Ratiyakorn Korkong $^{3} \cdot$ \\ Somkamon Ardsawang ${ }^{3}$. Sirapatsorn Ronglakorn ${ }^{3} \cdot$ Kanit Sananpanich $^{4} \cdot$ Siwat Suksri $^{3}$. \\ Athikhun Suwannakhan ${ }^{1,2}$ (1)
}

Received: 30 July 2021 / Accepted: 1 November 2021 / Published online: 13 November 2021

(c) The Author(s), under exclusive licence to Springer-Verlag France SAS, part of Springer Nature 2021

\begin{abstract}
Purpose Two most common variations of flexor pollicis longus include its accessory head and its connection with the flexor digitorum profundus of the index (Linburg-Comstock variation). In addition, while three-dimensional (3D) screening has widely been used in anatomical education, its use as reporting tool in anatomical research is still limited. The objective of this study is to report a previously unrecognized form of the accessory head of flexor pollicis longus, discuss the potential etiology of Linburg-Comstock variation, and pilot the 3D scanning of a large-scale anatomical structure.

Methods An unusual tendon slip was discovered during a routine dissection in the anterior compartment of the right forearm of a 54-year-old male cadaver. A 3D scanner was used to capture the surface topography of the specimen and an interactive portable document format (PDF) was created.

Results An anomalous tendon was found originating from the lateral aspect of the flexor digitorum profundus muscle. This variant tendon then inserted onto the medial surface of the flexor pollicis longus tendon before entering the carpal tunnel. The variation resembles a reverse form of Linburg-Comstock variation, because pulling this variant tendon resulted in simultaneous flexion of the interphalangeal joint of thumb.

Conclusion Surgeons should be aware of the reverse Linburg-Comstock variation, because it may not be detectable by the conventional provocative testing. Linburg-Comstock variation may be classified as an anatomical variant or a secondarily acquired condition depending on its type. Our demonstration of interactive 3D-PDF file highlights its potential use for delivering anatomical information in future cadaveric studies.
\end{abstract}

Keywords Flexor pollicis longus $\cdot$ Flexor digitorum profundus $\cdot$ Linburg-Comstock variation $\cdot$ Anatomical variations $\cdot 3 \mathrm{D}$ scanning $\cdot 3 \mathrm{D}$ visualization $\cdot$ Surface rendering

\section{Introduction}

Athikhun Suwannakhan

athikhun.suw@mahidol.edu

1 Department of Anatomy, Faculty of Science, Mahidol University, Bangkok, Thailand

2 In Silico and Clinical Anatomy Research Group (iSCAN), Department of Anatomy, Faculty of Science, Mahidol University, Bangkok, Thailand

3 Media Technology, King Mongkut's University of Technology Thonburi, Bangkok, Thailand

4 Department of Orthopedics, Faculty of Medicine, Chiang Mai University, Chiang Mai, Thailand
First described by Linburg and Comstock [15], Linburg-Comstock variation (LCV) is the connection between the tendon of flexor pollicis longus (FPL) and the flexor digitorum profundus of the index finger (FDP-I) which can be between the tendons themselves, their tendon sheaths or even muscle bellies [11]. This variation is present in around $21 \%$ (5.6-58.7\%) of the general population [23]. Individuals with LCV are able to simultaneously flex the distal interphalangeal joint of the index while actively flexing the distal phalange of the thumb [14]. Although this variation remains asymptomatic in most cases, it may cause disability and pain in some individuals, which may require surgical exploration 
and excision. In case the variation is symptomatic, the term "Linburg-Comstock syndrome" may be used to described the condition [23]. The LCV typically takes proximal origin from the FPL, which then distally joins the medial side of the FDP-I tendon. In rare cases, multiple FDP tendons may be involved including those of the middle and ring fingers $[15,25]$. The reverse form of the LCV, in other words, the LCV running from the FDP to the FPL has never been reported. Also, despite numerous studies [23] examining the anatomy and prevalence of LCV, the etiology of all three types of LCV has never been considered.

Three-dimensional (3D) scanning is increasing becoming an integrable part of anatomy education, because 3D anatomical models are more educationally effective in terms of spatial and factual knowledge [24]. The scanned models can be provided electronically, which allows users to move, rotate and magnify in the closest way possible to real specimens [1,8]. One fundamental benefit of electronic 3D models is they can provide alternative resources to learners who may otherwise not have access to original specimens, especially amid the COVID-19 pandemic where online anatomy courses are inevitable [7]. These models can be embedded into a portable document format file (PDF) creating interactive 3D-PDF files [1]. Although the use of these interactive 3D-PDF files is not yet widely adopted, a recent review identified embedded 3D content in more than 200 articles across various disciplines either as a dedicated figure or as an external link [17]. Despite potential opportunities that 3D-PDF technology offers especially in anatomical education and other research fields, its potential for presenting interactive anatomical information in the scope of anatomical research has been little explored.

In the present study, we report a unique tendon slip running from the FDP muscle belly to the FPL tendon. This unique tendinous variant resembles a reverse configuration of the LCV. Possible etiology of the LCV is also discussed in detail. In addition, an interactive 3D-PDF of our finding was created to illustrate the potential use of 3D-PDF for presenting anatomical information in future dissection-based anatomical studies.

\section{Materials and methods}

\section{Dissection and preparation}

During a routine dissection at the Department of Anatomy, Faculty of Science, Mahidol University, an anomalous tendon was found in a right forearm a 54-year-old male cadaver (Fig. 1). Its morphology, origin and insertion were carefully inspected. There were no other variations in both limbs. No other notable anomalies were found in the body. The dissection was carried out by the authors. Informed consent of body donation was obtained from the donor in advance, and no ethical approval was required for this type of study. Still images and video of the present case were captured using iPhone 12 Pro (Apple Inc., California, USA). Prior to $3 \mathrm{D}$ scanning, the cadaver was placed on the dissection table in the supine position with its right limb abducted at a 45-degree angle. Before scanning, glycerin preservative was wiped off from the specimen to create reflection-free surface. Dark green vinyl fabric was used as background.

\section{Three-dimensional scanning and creation of 3D-PDF}

The model of the 3D scanner was Einscan Pro purchased from Shining 3D Tech (Hangzhou, China). This scanner has a single shot accuracy of $0.05 \mathrm{~mm}$. The surface topography of the anterior compartment of the right upper limb was captured using fixed method where the scanner was mounted from above at a 45-degree angle with a distance around $12 \mathrm{~cm}$ from the specimen. No additional lighting was used. The light-emitting diode (LED) patterns were emitted onto the specimen, while sensors detected the edge of the patterns. With trigonometric triangulation, the distance from the light source to the sensor can be calculated to create a 3D construct. The 3D mesh was processed using the manufacturer's software. Any unwanted parts were erased at this stage. The resulting model was then converted to polygon mesh and was subsequently exported as an Object File (OBJ). The model underwent further editing using Blender (Blender Foundation, Amsterdam) before being exported as a Filmbox format (FBX), which was then further converted to Universal 3D format (U3D) using Filestar (Filestar AB, Saltsjö-Boo, Sweden). Adobe Acrobat Pro (Adobe Inc., California, USA) was used to create a rotatable PDF (Fig. 2 and Supplementary File 1).

\section{Results}

An anomalous tendon slip was observed in the anterior compartment in the right forearm (Fig. 1). This tendon variant arose from the radial side of the FDP muscle belly. This variant became tendinous around mid-forearm, and travelled distally between FDP and the FPL tendons (Fig. 1c). The variant tendon was located in the deep anterior compartment of forearm and was positioned between the radial side of the FDP tendon and the ulnar side of the FPL tendon. The tendon width was approximately $2-3 \mathrm{~mm}$ throughout its length. The tendon was then inserted onto the medial surface of the FPL tendon before entering the carpal tunnel. Pulling the variant tendon produced flexion of the interphalangeal joint of the thumb (Supplementary Video 1). The lateral portion of the FDP muscle belly also partly originated from the proximal one thirds of the radius. The FDP was innervated 

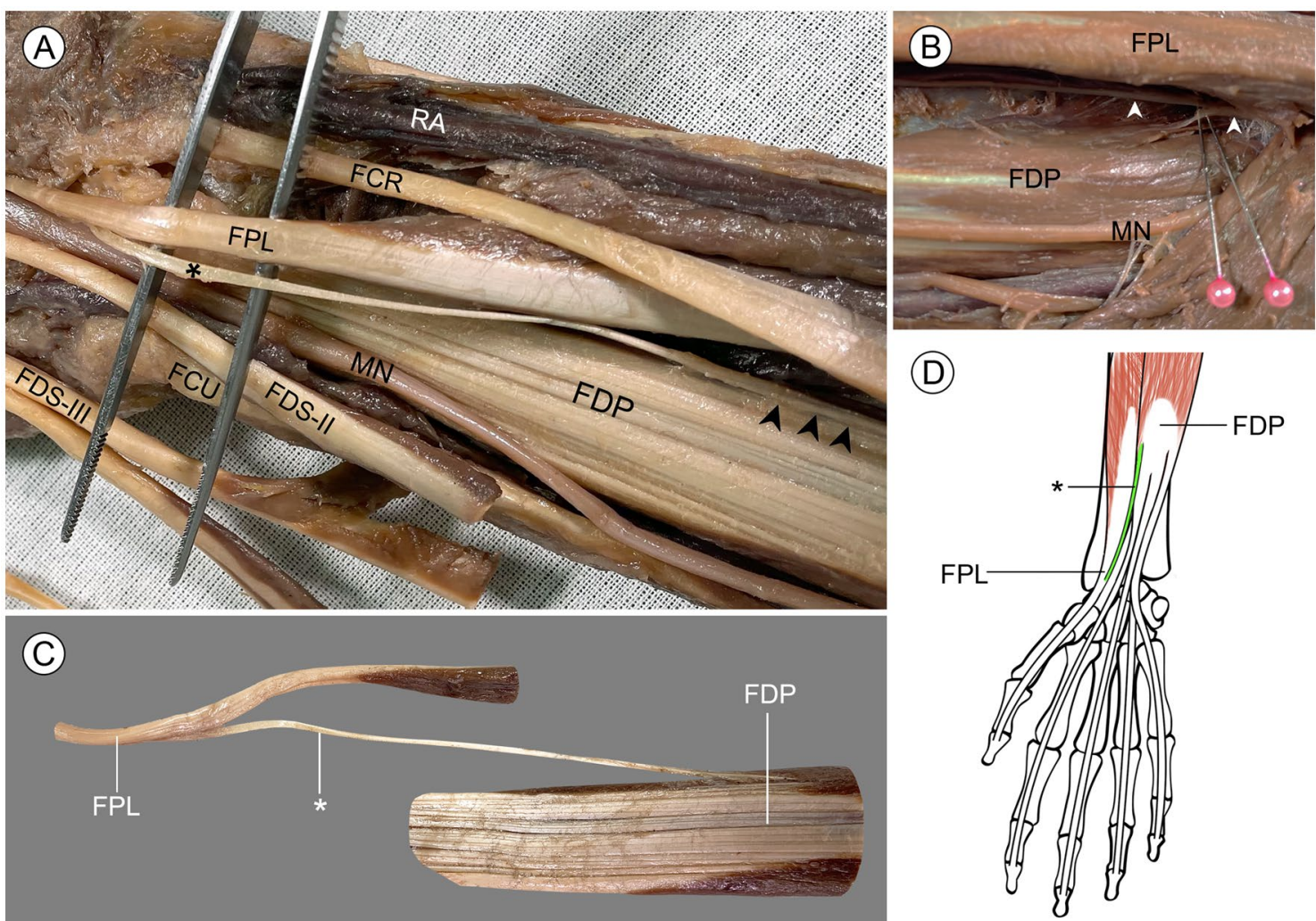

Fig. 1 Dissection (a-c) and illustrative diagram (d) showing a variant tendon slip $\left(^{*}\right)$ originating from the FDP in the anterior compartment of forearm of the right limb of a 54-year-old male cadaver. FPL flexor pollicis longus, $F D P$ flexor digitorum profundus, $M N$ median nerve, $F C R$, flexor carpi radialis, FCU flexor carpi ulnaris, FDS-II/
III flexor digitorum superficialis of second and third digits, $R A$ radial artery. Black arrow heads indicate the origin of variant tendon from the FDP muscle belly. White arrow heads and the pink pins indicate the innervation from the anterior interosseous nerve over the origin of FDP from radius (colour figure online)

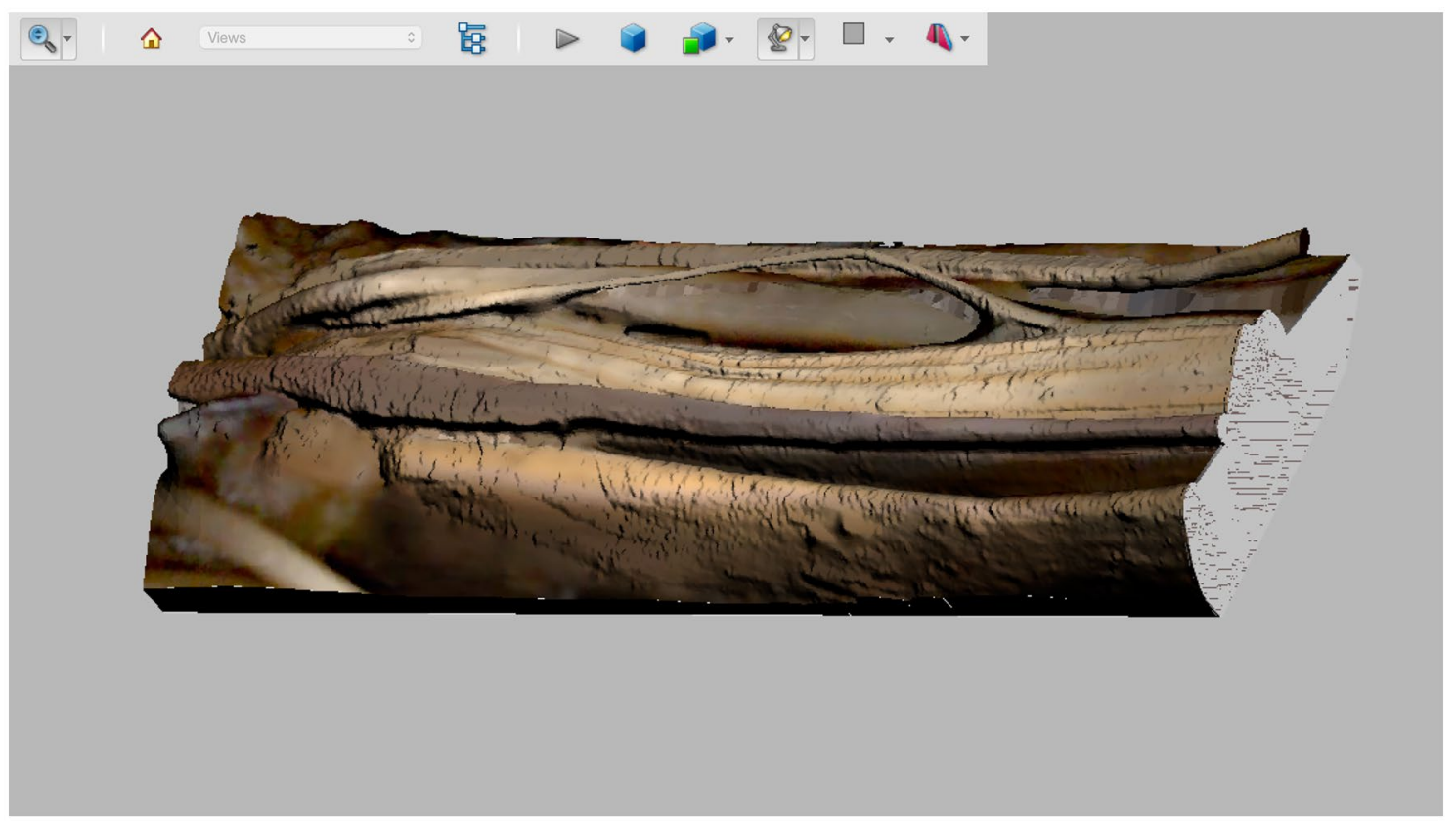

Fig. 2 The 3D-PDF demonstrating the variant tendon. Rotatable 3D-PDF is available as supplementary material 
a single branch from the anterior interosseous nerve of the median nerve (Fig. 1b). Rotatable 3D-PDF (Fig. 2) of the specimen is available in the supporting information (Supplementary File 1).

\section{Discussion}

The present study reports an unusual tendinous slip running from the FDP muscle belly to the FPL tendon. To our knowledge, no similar variant has been reported previously. Variations of the FDP tendons are rare and were only described by a few studies. Jain et al. [9] reported an accessory FDP tendon which bifurcated before joining the FDP tendons of the index and middle fingers. Macalister [16] described several variations of the FDP tendons such as an additional FDP slip to the FDS tendon of the little or index fingers, fourth lumbrical arising from the FDP tendon, its division into five slips, and a tendon joining the indicial part of the FDP similar to the LCV.

While this variant appeared to be an accessory tendon slip of the FDP, it may alternatively be viewed as an accessory head of the FPL or more commonly known as Gantzer's muscle. Even though Gantzer's muscle is typically associated with the FPL, it is rarely associated with the flexor digitorum superficialis (FDS) or FDP [10, 19]. According to a meta-analysis [19], the accessory head of FPL takes origin from the medial epicondyle of the humerus (44\%), coronoid process of the ulna $(26 \%)$ or both $(16 \%)$. In less than $1 \%$ of total cases, the accessory FPL originates from the flexor digitorum superficialis or muscular fascia [19]. The accessory head of FPL inserts onto the FPL tendon in $95 \%$ of all cases, while in the remaining 5\%, it joins the FDP. An unrecognized structure from the FPL muscle belly accompanied by an accessory head of FPL has recently been found inserting into the distal FPL tendon [28]. Despite numerous studies investigating the variations of the FPL, an accessory tendon slip of FPL from the FDP as presented in our study has never been reported.

The tendinous variant presented in this study resembles a reverse configuration of the LCV. Generally, the LCV proximally arises from the FPL tendon before joining the
FDP of the index finger distally. The connection can occur between the tendons themselves, the tendon sheaths or the muscle bellies [11]. Clinical test using three different thumb flexion motions is performed to detect the conventional form of LCV running from the FPL to the FDP-I [14]. However, this test was not designed to test the reversed LCV running from the FDP to FPL. Typically, when there is a simultaneous flexion of the distal interphalangeal joint of the index upon the thumb flexion, the test is accepted as positive. In case of reversed LCV, the aforementioned test may yield a fault negative as demonstrated in the present study (Supplementary Video 1), because the connection would simply lax without producing flexion of the DIP joint of the index finger. To account for the possibility of a reversed LCV, we propose that the subject is asked to actively flex the DIP joint of the remaining fingers especially the index while the thumb is fully extended. This test may be recommended when there is restricted range of motion of the thumb and the conventional test for LCV is negative. Further in vivo studies should also be conducted to examine the presence of reversed LCV in wider population groups.

Despite a large body of research on LCV, its etiology remains inconclusive. While many studies recognized LCV as a vestigial structure [2], some believe it is secondarily acquired [21]. In our view, it is most probable that the etiology of LCV is type-dependent (Table 1). According to its histological analysis [27], the fibrous LCV contains dense irregular connective tissue with numerous nuclei corresponding to synovial hyperplasia. Such observation is in line with a recent clinical case reported by Tasche et al. [21], whereby the adhesion between FPL and FDP-I developed following a trauma, producing a manifestation similar to that of the LCV [21]. These findings lead to a plausible conclusion that fibrous LCV might be a secondarily acquired structure.

Tendinous LCV may be a vestigial structure representing the connection between FDP-I and FDP of the thumb in some non-human primates. True FPL with distinct muscle belly is only present in humans and hylobatids. Other non-human primates, however, have ancestral configuration whereby FDP inserts to the distal phalanges of all digits. Interestingly, in a dissection of young infant $H$. gabriellae
Table 1 Classification of Linburg-Comstock variation and its possible etiology

\begin{tabular}{lll}
\hline Type & Explanation & Possible etiology \\
\hline Fibrous & Adhesion between FPL and FDP-I synovial sheaths [22] & Acquired \\
& Adhesion between FPL tendon sheath and the radial bursa & Acquired \\
& containing the FDP-I tendon [7] & \\
Tendinous & Vestigial form of FDP to the index [3] & Vestigial \\
& Accessory tendon slip from FDP to FPL (present study) & Variation \\
Musculotendinous & Accessory first lumbrical muscle [27] & Variation \\
& Vestigial form of FDP to the index [3] & Vestigial \\
\hline
\end{tabular}


species, a tendinous connection between FDP tendon that goes to digit one and the FDP tendon that goes to the index finger [3], similar to tendinous LCV. During the course of evolution, there was a selection pressure towards the independent functionality of the thumb. This connection reflects the ancestral configuration where FPL and FDP were formed as a single structure [5]. All these data lead to a possible conclusion that tendinous LCV might be an evolutionary remnant rather than a secondarily acquired structure.

Musculotendinous type of LCV may be classified as an accessory anatomical variant or vestigial structure. Muscles of the upper limb originate from the somatic mesoderm around the fourth week of development. The flexor mass further develops into superficial and deep layers [6]. The deep layer gives rise to the FDP, FDS, FPL and the lumbricals. The incomplete cleavage of these layers is believed to be the cause of accessory or variant forms of these muscles [10]. The case of musculotendinous LCV presented in Yurasakpong et al. [27] resembles to a great extent a variant lumbrical originating from the FPL, similar to previous reports [12, 13, 27]. With regards to its possible etiology, it was speculated that variants in the adult stage were found to be commonly expressed during the embryonic period [4], for example, the dorsometacarpales $[4,26]$, a group of primitive digital extensors. Recently, we discovered a variant extensor muscle which extended to all five digits in the deep extensor compartment of forearm [20], similar to the extensor digitorum profundus complex found in non-human primate species [18]. These findings suggest the possibility that the musculotendinous LCV could be an accessory anatomical variant or a vestigial structure.

To our knowledge, the present work is the first study that uses 3D-PDF as a reporting tool in anatomical research. In anatomical research, 3D imaging allows for a more complete and interactive representation of the specimen. It also ensures transparency of the reported finding, because 3D models are more difficult to manipulate when compared with conventional 2D images. There are two methods of 3D scanning including fixed scanning and handheld scanning. For fixed scanning, the scanner is mounted from a higher position, while the specimen is placed on a flat rotatable surface. This method was previously used to build 3D digital replica of fish chondrocranium and an amphibian skeleton with good retention of anatomical features and minor loss of fidelity [22]. Fixed scanning generally offers higher resolution but requires longer time than handheld scanning. Markers, or small stickers recognizable by the scanner, could be utilized as reference points to ensure accuracy in the measurement if handheld scanning was used. In this study, only the anterior side of forearm was scanned, because it was not possible to keep the structures of a thoroughly dissected specimen in place. Preservatives such as glycerin may be problematic because of its glossy nature which causes unwanted reflection from the specimen itself. In our study, Adobe Acrobat Pro software was used to create an interactive 3D-PDF. Alternatively, 3D-PDFs could be created in latex using media9 package as recently described by Azkue [1].

While 3D scanning appears to be a promising reporting tool in anatomical research, it is of course associated with a few limitations. 3D scanners are costly and may not be viewed as a necessity for most institutions. Qlone, a simpler and free cellphone application, has recently been used to produce 3D skeletal models for anatomy teaching, although improvements are still needed to better visualize deeply located structures [8]. Also, the unprocessed 3D images have to undergo further editing and format conversion that requires several software applications. Highly complex structures of the upper limb such as nerves and vessels could not clearly be delimited at this stage. Further studies are also needed to optimize the scanning to achieve higher resolution, accuracy and aesthetic quality, particularly for large-scale structures.

In conclusion, we report an anomalous tendinous connection running from the FDP muscle belly to the FPL tendon, which resembles a reverse form of the LCV. Despite future improvements, our study indicates that 3D-PDF technology is a promising tool for presenting interactive anatomical information and offers a potential new standard for cadaveric studies in the future.

Supplementary Information The online version contains supplementary material available at https://doi.org/10.1007/s00276-021-02858-8.

Acknowledgements The authors express our deepest gratitude to the body donor and their families.

Author contributions WP, NK, and AS: project development, data analysis, and manuscript writing/editing. WP, NK, KS, and LY: data analysis and manuscript writing/editing. RK, SA SR and SS: data acquisition. WP, LY, SS and AS: project development, data analysis, and manuscript writing/editing.

Funding None.

\section{Declarations}

Conflict of interest The authors declare no conflict of interest.

\section{References}

1. Azkue JJ (2021) Embedding interactive, three-dimensional content in portable document format (PDF) to deliver gross anatomy information and knowledge. Clin Anat 34:919-933. https://doi. org/10.1002/ca.23755

2. Bulut T, Tahta M, Ozturk T, Zengin EC, Ozcan C, Sener M (2017) Linburg-Comstock: is overuse an etiological factor? Plast Surg 25:268-271. https://doi.org/10.1177/2292550317731763 
3. Diogo R, Richmond BG, Wood B (2012) Evolution and homologies of primate and modern human hand and forearm muscles, with notes on thumb movements and tool use. J Hum Evol 63:64 78. https://doi.org/10.1016/j.jhevol.2012.04.001

4. Diogo R, Siomava N, Gitton Y (2019) Development of human limb muscles based on whole-mount immunostaining and the links between ontogeny and evolution. Development 146:dev180349. https://doi.org/10.1242/dev.180349

5. Diogo R, Wood B (2011) Soft-tissue anatomy of the primates: phylogenetic analyses based on the muscles of the head, neck, pectoral region and upper limb, with notes on the evolution of these muscles. J Anat 219:273-359. https://doi.org/10.1111/j. 1469-7580.2011.01403.x

6. El Domiaty MA, Zoair MM, Sheta AA (2008) The prevalence of accessory heads of the flexor pollicis longus and the flexor digitorum profundus muscles in Egyptians and their relations to median and anterior interosseous nerves. Folia Morphol 67:63-71

7. Iwanaga J, Loukas M, Dumont AS, Tubbs RS (2021) A review of anatomy education during and after the COVID-19 pandemic: revisiting traditional and modern methods to achieve future innovation. Clin Anat 34:108-114. https://doi.org/10.1002/ca.23655

8. Iwanaga J, Terada S, Kim H-J, Tabira Y, Arakawa T, Watanabe K, Dumont AS, Tubbs RS (2021) Easy three-dimensional scanning technology for anatomy education using a free cellphone app. Clin Anat 34:910-918. https://doi.org/10.1002/ca.23753

9. Jain S, Kumar S, Herswani I (2021) A case report: rare variation of an extra musculo-tendon with two slips of flexor digitorum profundus muscle. J Ayurveda 15:109-112

10. Jones M, Abrahams PH, Sañudo JR, Campillo M (1997) Incidence and morphology of accessory heads of flexor pollicis longus and flexor digitorum profundus (Gantzer's muscles). J Anat 191:451455. https://doi.org/10.1046/j.1469-7580.1997.19130451.x

11. Kirchberger MC, Hahn P, Unglaub F, Leclère F, Spies C (2015) The Linburg-Comstock phenomenon: a review. Handchir Mikrochir Plast Chir 47:58-61

12. Koizumi M, Kawai K, Honma S, Kodama K (2002) Anomalous lumbrical muscles arising from the deep surface of flexor digitorum superficialis muscles in man. Ann Anat 184:387-392. https:// doi.org/10.1016/s0940-9602(02)80062-0

13. Kumar A, Mishra A, Goswami D, Ganesh E (2014) Morphological cariations in lumbricals of upper limb: a cadaveric study. Anat Physiol 5:1000165. https://doi.org/10.4172/2161-0940.1000165

14. Leyva KA, Laslovich S, Roberts T, Kane E (2021) An investigation into the reliability and methodology of the Linburg-Comstock anomaly clinical test. J Hand Ther 34:433-438. https://doi. org/10.1016/j.jht.2020.03.015

15. Linburg RM, Comstock BE (1979) Anomalous tendon slips from the flexor pollicis longus to the flexor digitorum profundus. $\mathrm{J}$ Hand Surg Am 4:79-83. https://doi.org/10.1016/s0363-5023(79) 80110-0

16. Macalister A (1875) Additional observations on muscular anomalies in human anatomy (third series) with a catalogue of the principal muscular variations Hitherto published. Trans R Ir Acad $25: 1-134$
17. Newe A, Becker L (2018) Three-dimensional portable document format (3D PDF) in clinical communication and biomedical sciences: Systematic review of applications, tools, and protocols. JMIR Med Inform 6:e10295. https://doi.org/10.2196/10295

18. Novikova MA, Panyutina AA (2017) Structural diversity of the extensor digitorum profundus muscle complex in platyrrhini. Folia Primatol 88:274-292. https://doi.org/10.1159/000478524

19. Roy J, Henry BM, Pękala PA, Vikse J, Ramakrishnan PK, Walocha JA, Tomaszewski KA (2015) The prevalence and anatomical characteristics of the accessory head of the flexor pollicis longus muscle: a meta-analysis. PeerJ 3:e1255. https://doi.org/10.7717/ peerj. 1255

20. Suwannakhan A, Nontunha N, Meemon K (2020) Complete extensor digitorum profundus complex: a deep hand extensor muscle to the medial four digits. Surg Radiol Anat 42:935-938. https:// doi.org/10.1007/s00276-020-02439-1

21. Tasch C, Djedovic G, Haim A, Zimmermann R (2018) Secondary Linburg-Comstock syndrome: a case report. Eur J Plast Surg 41:355-358. https://doi.org/10.1007/s00238-017-1359-y

22. Thomas DB, Hiscox JD, Dixon BJ, Potgieter J (2016) 3D scanning and printing skeletal tissues for anatomy education. J Anat 229:473-481. https://doi.org/10.1111/joa.12484

23. Yammine K, Erić M (2018) Linburg-Comstock variation and syndrome. A meta-analysis. Surg Radiol Anat 40:289-296. https:// doi.org/10.1007/s00276-017-1957-1

24. Yammine K, Violato C (2015) A meta-analysis of the educational effectiveness of three-dimensional visualization technologies in teaching anatomy. Anat Sci Educ 8:525-538. https://doi.org/10. 1002/ase. 1510

25. Yoon HK, Kim CH (2013) Linburg-Comstock syndrome involving four fingers: a case report and review of the literature. J Plast Reconstr Aesthet Surg 66:1291-1294. https://doi.org/10.1016/j. bjps.2012.12.032

26. Yurasakpong L, Diogo R, Chaiyamoon A, Meemon K, Suwannakhan A (2020) Extensor indicis radialis and extensor medii proprius associated with an unknown fibromuscular slip: a case report. SN Compr Clin Med 2:2456-2459. https://doi.org/10. 1007/s42399-020-00545-0

27. Yurasakpong L, Meemon K, Suwannakhan A (2018) LinburgComstock variation: histoanatomy and classification of the connection between flexor pollicis longus and flexor digitorum profundus to the index finger. Surg Radiol Anat 40:297-301. https:// doi.org/10.1007/s00276-018-1985-5

28. Zielinska N, Szewczyk B, Tubbs RS, Olewnik Ł (2021) Coexistence of two accessory flexor pollicis longus heads or coexistence of two-headed flexor pollicis longus with an unrecognized anatomical structure? Surg Radiol Anat 43:763-769. https://doi.org/ 10.1007/s00276-021-02721-w

Publisher's Note Springer Nature remains neutral with regard to jurisdictional claims in published maps and institutional affiliations. 